\title{
Decoherence of Atomic Gases in Largely Detuned Laser Fields
}

\author{
Karl-Peter Marzlin \\ Fachbereich Physik der Universität Konstanz, Postfach 5560 M674, D-78457 Konstanz, Germany
}

(October 31, 2018)

\begin{abstract}
We study theoretically the decoherence of a gas of bosonic atoms induced by the interaction with a largely detuned laser beam. It is shown that for a standing laser beam decoherence coincides with the single-particle result. For a running laser beam many-particle effects lead to significant modifications.
\end{abstract}

32.80.-t, 03.65.Yz, 03.75.Fi

In experiments with atomic Bose-Einstein condensates [1,2] the trap which is used to isolate the atoms from the environment plays an important role for the physical behaviour of the system. In a magnetic trap usually only atoms with a specific magnetic hyperfine quantum number are stored. An optical trap [3] can be used to confine atoms in all Zeeman sublevels. However, in order to preserve the atomic coherence spontaneous emission of photons must be avoided. A large detuning $\Delta$ of the laser beams providing the trap field reduces the excitation probability for atoms and thus reduces the number of spontaneously emitted photons. At the same time, a relatively strong intensity of the laser beams, and correspondingly a large Rabi frequency $\Omega(\mathbf{x})$, ensures that the laser beams' effect is still large enough to produce a strong potential for the atoms.

The aim of this paper is to examine light-induced decoherence of a gas of ultracold atoms in an optical trap and to deduce the influence of atomic many-particle properties on this decoherence. To do so we study solutions to a master equation for reduced Heisenberg operators describing a quantum field of bosonic two-level atoms interacting with a detuned laser beam and the vacuum fluctuations of the electromagnetic field. The technique of reduced Heisenberg operators has previously been employed by Nemoto and Shibata [4] and allows an elegant description of decoherence in a many-particle context. After the derivation of the master equation we will adiabatically eliminate the excited state to get an effective master equation for the atomic internal ground state. The main result of the paper is that many-particle effects lead to a nonlocal modification of light-induced decoherence that vanishes for laser fields with spatially homogeneous phase (e.g., a standing laser wave) but makes substantial contributions for a running laser wave.

Derivation of the master equation: We consider a general system that is described by the direct product of two Hilbert spaces $\mathcal{H}_{S}$ and $\mathcal{H}_{R}$, where $\mathcal{H}_{S}$ describes the open system that we are interested in, and $\mathcal{H}_{R}$ describes the reservoir to which the system is coupled. It is our aim to find a master equation describing the evolution of reduced Heisenberg operators for the system only. We define a reduced Heisenberg operator $\hat{R}_{S}$ by the relation

$$
\hat{R}_{S}(t):=\operatorname{Tr}_{R}\left\{\hat{\rho}_{R} \hat{R}(t)\right\},
$$

where $\hat{R}(t)$ is the original Heisenberg operator which acts on $\mathcal{H}_{S} \otimes \mathcal{H}_{R}$ and $\hat{\rho}_{R}$ is the density matrix describing the state of the reservoir. It is thereby assumed that the (time-independent) density matrix can be written in the form $\hat{\rho}=\hat{\rho}_{S} \otimes \hat{\rho}_{R}$ so that there are initially no correlations between the system and the reservoir. The physical significance of the reduced Heisenberg operator is that it allows to describe accurately any measurement involving the system only, i.e.,

$$
\operatorname{Tr}_{S \otimes R}\left\{\hat{\rho} \hat{F}_{S} \hat{R}(t) \hat{F}_{S}^{\prime}\right\}=\operatorname{Tr}_{S}\left\{\hat{\rho}_{S} \hat{F}_{S} \hat{R}_{S}(t) \hat{F}_{S}^{\prime}\right\}
$$

for any two operators $\hat{F}_{S}$ and $\hat{F}_{S}^{\prime}$ acting on $\mathcal{H}_{S}$ only.

To derive a master equation for $\hat{R}_{S}(t)$ we employ Zwanzig's method by using that $\mathcal{P} \hat{R}:=\operatorname{Tr}_{R}\left\{\hat{\rho}_{R} \hat{R}\right\} \otimes \mathbf{1}_{R}$ is a projection superoperator fulfilling $\mathcal{P}^{2}=\mathcal{P}$. We start from the Heisenberg equation-of-motion

$$
i \hbar \partial_{t} \hat{R}(t)=[\hat{R}(t), \hat{H}]=: \mathcal{L} \hat{R}(t),
$$

where $\mathcal{L}$ is the Liouville superoperator. The Hamiltonian in Heisenberg picture is explicitely time-independent if it is written in terms of Schrödinger picture operators. Introducing $\mathcal{Q}:=1-\mathcal{P}$ and following the lines of Ref. [5] one can derive the master equation for the reduced Heisenberg operator,

$$
\begin{aligned}
i \hbar \partial_{t} \hat{R}_{S}(t)= & \mathcal{P} \mathcal{L} \mathcal{P} \hat{R}_{S}(t) \\
& -\frac{i}{\hbar} \mathcal{P} \mathcal{L} \int_{t_{0}}^{t} d t^{\prime} U_{Q Q}\left(t^{\prime}\right) \mathcal{Q L P} \hat{R}_{S}\left(t-t^{\prime}\right)
\end{aligned}
$$

In the derivation it was assumed that the inital Heisenberg operator $\hat{R}(0)$ acts on the system only so that $\mathcal{Q} \hat{R}(0)=0$. The superoperator $U_{Q Q}\left(t^{\prime}\right):=$ $\exp \left\{-i t^{\prime} \mathcal{Q} \mathcal{L} \mathcal{Q} / \hbar\right\}$ describes the unitary evolution in $\mathcal{Q}$ subspace.

This expression can be further simplified if the Liouvillean is written in the form $\mathcal{L}=\mathcal{L}_{S}+\mathcal{L}_{R}+\mathcal{L}_{\text {int }}$, where $\mathcal{L}_{S}$ and $\mathcal{L}_{R}$ act solely on the system and the reservoir, respectively, and $\mathcal{L}_{\text {int }}$ describes the interaction. It is then easy to show that $\mathcal{Q} \mathcal{L} \mathcal{P}=\mathcal{Q} \mathcal{L}_{\text {int }} \mathcal{P}$ and, if the reservoir is in a stationary state $\left(\left[\hat{H}_{R}, \hat{\rho}_{R}\right]=0\right), \mathcal{P} \mathcal{L} \mathcal{Q}=\mathcal{P} \mathcal{L}_{\text {int }} \mathcal{Q}$. 
Expanding Eq. (4) to second order in $\mathcal{L}_{\text {int }}$ we can replace $U_{Q Q}\left(t^{\prime}\right)$ by $\exp \left\{-i t^{\prime}\left(\mathcal{L}_{S}+\mathcal{L}_{R}\right) / \hbar\right\}$. Performing a Markov approximation $\left(\hat{R}_{S}\left(t-t^{\prime}\right) \approx U_{P P}^{-1}\left(t^{\prime}\right) \hat{R}_{S}(t)\right.$ under the integral) then results in the final form of the master Equation in Heisenberg picture,

$$
i \hbar \partial_{t} \hat{R}_{S}(t) \approx \mathcal{L}_{S} \hat{R}_{S}(t)-\frac{i}{\hbar} \mathcal{P} \mathcal{L}_{\mathrm{int}} \mathcal{Q} \int_{0}^{\infty} d t^{\prime} \mathcal{L}_{\mathrm{int}}\left(t^{\prime}\right) \hat{R}_{S}(t)
$$

with $\mathcal{L}_{\text {int }}\left(t^{\prime}\right) \hat{R}_{S}=\left[\hat{R}_{S}, \hat{H}_{\text {int }}\left(t^{\prime}\right)\right]$ and $\hat{H}_{\text {int }}\left(t^{\prime}\right) \quad:=$ $\exp \left\{-i t^{\prime}\left(\mathcal{L}_{S}+\mathcal{L}_{R}\right) / \hbar\right\} \hat{H}_{\text {int }}$.

Decoherence of the atomic field operator: The formalism of the previous section will now be applied to the problem of spontaneous emission in a system of bosonic two-level atoms. In this case the degrees-offreedom of the electromagnetic field play the role of a reservoir to which the atoms are coupled. Since it is known that usually the center-of-mass motion of the atoms has only a small influence on the spontaneous emission rate [6,7] we neglect it alltogether and use the atomic Hamiltonian

$$
\hat{H}_{S}=\int d^{3} x \hat{\Psi}_{e}^{\dagger}(\mathbf{x}) \hbar \omega_{0} \hat{\Psi}_{e}(\mathbf{x})
$$

where $\omega_{0}$ is the resonance frequency and $\hat{\Psi}_{i}(\mathbf{x})$ are the quantum field operators for excited $(i=e)$ and groundstate $(i=g)$ atoms, respectively. The Hamiltonian of the reservoir is given by

$$
\hat{H}_{R}=\frac{\varepsilon_{0}}{2} \int d^{3} x\left\{\hat{\mathbf{E}}^{2}+c^{2} \hat{\mathbf{B}}^{2}\right\}
$$

and the coupling between the system of two-level atoms and the reservoir is described by the electic-dipole coupling in rotating-wave approximation,

$$
\begin{array}{r}
\hat{H}_{\mathrm{int}}\left(t^{\prime}\right)=\int d^{3} x\left\{\hat{\mathbf{E}}^{(+)}\left(\mathbf{x}, t^{\prime}\right) \hat{\mathbf{P}}^{(-)}(\mathbf{x}) e^{i \omega_{0} t^{\prime}}+\right. \\
\left.\hat{\mathbf{E}}^{(-)}\left(\mathbf{x}, t^{\prime}\right) \hat{\mathbf{P}}^{(+)}(\mathbf{x}) e^{-i \omega_{0} t^{\prime}}\right\},
\end{array}
$$

with $\hat{\mathbf{E}}^{(+)}\left(\mathbf{x}, t^{\prime}\right)$ being the positive-frequency part of the electric field in interaction picture and $\hat{\mathbf{P}}^{(+)}(\mathbf{x}):=$ $\mathbf{d} \hat{\Psi}_{e}(\mathbf{x}) \hat{\Psi}_{g}^{\dagger}(\mathbf{x})$ is the positive-frequency part of the polarization operator.

Under the assumption that the initial state of the electromagnetic field is the vacuum $|0\rangle$ we can insert the various Hamiltonians into Eq. (5) to derive

$$
i \hbar \partial_{t} \hat{R}_{S}(t)=\left[\hat{R}_{S}(t), \hat{H}_{S}\right]-i \hbar \mathcal{L}_{D} \hat{R}_{S}(t),
$$

with the decoherence Liouvillean

$$
\begin{aligned}
\mathcal{L}_{D} \hat{R}:=\int & d^{3} x d^{3} x^{\prime}\left\{\left[\hat{R}, P_{i}^{(-)}(\mathbf{x})\right] P_{j}^{(+)}\left(\mathbf{x}^{\prime}\right) T_{i j}\left(\mathbf{x}, \mathbf{x}^{\prime}\right)+\right. \\
& \left.P_{i}^{(-)}(\mathbf{x})\left[P_{j}^{(+)}\left(\mathbf{x}^{\prime}\right), \hat{R}\right] T_{i j}^{*}\left(\mathbf{x}, \mathbf{x}^{\prime}\right)\right\}
\end{aligned}
$$

The quantity $T_{i j}$ can be calculated by standard methods (see, e.g., Refs. [8.9]) and is given by

$$
\begin{aligned}
T_{i j}\left(\mathbf{x}, \mathbf{x}^{\prime}\right):= & \int_{0}^{\infty} d t^{\prime}\left\langle 0\left|E_{i}^{(+)}\left(\mathbf{x}, t^{\prime}\right) E_{i}^{(-)}\left(\mathbf{x}^{\prime}, 0\right)\right| 0\right\rangle \\
= & \int_{0}^{\infty} \frac{d \omega \omega^{3}}{4 \pi^{2} \hbar \varepsilon_{0} c^{3}}\left(\pi \delta\left(\omega-\omega_{0}\right)-i \frac{P}{\omega-\omega_{0}}\right) \times \\
& {\left[\delta_{i j}\left(j_{0}\left(\frac{\omega r}{c}\right)-\frac{j_{1}\left(\frac{\omega r}{c}\right)}{\frac{\omega r}{c}}\right)+\right.} \\
& \left.\hat{\mathbf{r}}_{i} \hat{\mathbf{r}}_{j}\left(\frac{3 j_{1}\left(\frac{\omega r}{c}\right)}{\frac{\omega r}{c}}-j_{0}\left(\frac{\omega r}{c}\right)\right)\right] .
\end{aligned}
$$

In the last expression we have defined $r:=\left|\mathbf{x}-\mathbf{x}^{\prime}\right|$ and $\hat{\mathbf{r}}:=\left(\mathbf{x}-\mathbf{x}^{\prime}\right) / r$.

It is not hard to see that the imaginary part of $T_{i j}$ leads only to a (non-local) modification of the Hamiltonian $\hat{H}_{S}$ and therefore does not lead to decoherence effects. Since it is the latter which we are interested in we will omit the imaginary part. Doing so and inserting the definition of the polarization operator into Eq. (10) results in

$$
\begin{aligned}
\mathcal{L}_{D} \hat{R}= & \frac{3 \gamma_{0}}{4} \int d^{3} x d^{3} x^{\prime}\left\{\left[\hat{R}, \Psi_{e}^{\dagger}(\mathbf{x}) \Psi_{g}(\mathbf{x})\right] \Psi_{g}^{\dagger}\left(\mathbf{x}^{\prime}\right) \Psi_{e}\left(\mathbf{x}^{\prime}\right)+\right. \\
& \left.\Psi_{e}^{\dagger}(\mathbf{x}) \Psi_{g}(\mathbf{x})\left[\Psi_{g}^{\dagger}\left(\mathbf{x}^{\prime}\right) \Psi_{e}\left(\mathbf{x}^{\prime}\right), \hat{R}\right]\right\} J(r, \theta)
\end{aligned}
$$

Here $\gamma_{0}:=|\mathbf{d}|^{2} \omega_{0}^{3} /\left(3 \pi \hbar \varepsilon_{0} c^{3}\right)$ is the single-atom spontaneous emission rate and the function $J(r, \theta)$ is defined as

$$
J(r, \theta):=\sin ^{2}(\theta) j_{0}\left(\frac{\omega_{0} r}{c}\right)+\frac{j_{1}\left(\frac{\omega_{0} r}{c}\right)}{\frac{\omega_{0} r}{c}}\left(3 \cos ^{2}(\theta)-1\right),
$$

where $\theta$ is the angle between $\mathbf{d}$ and $\hat{\mathbf{r}}$. In the context of master equations for reduced density matrices the decoherence term (12) is well known [10,11] and has recently been applied to study loading 12 and condensation 13] of a condensate in laser light.

Eq. (12) will be the starting point of our investigation on light-induced decoherence in many-atom systems. Before doing so we point out that the master equations Eq. (12) and (10) are very similar to corresponding equations for the reduced density matrix in Schrödinger picture. However, there is a striking difference: For the reduced density matrix $\hat{\rho}_{S}$ the Liouvillean for spontaneous emission includes terms in which the operators $\hat{\mathbf{P}}^{(+)}(\mathbf{x})$ and $\hat{\mathbf{P}}^{(-)}(\mathbf{x})$ appear to the left and to the right of the density matrix, respectively. This ordering is important as such a term describes the incoherent de-excitation of an atom, while the opposite ordering $\hat{\mathbf{P}}^{(+)}(\mathbf{x}) \hat{\rho}_{S} \hat{\mathbf{P}}^{(-)}(\mathbf{x})$ would describe an incoherent excitation of an atom. But it is exactly the latter type of ordering which appears in Eq. (10). The reason is that Eq. (10) does not describe the evolution of the density matrix in Schrödinger picture 
but that of an operator in the Heisenberg picture. When applied to a Heisenberg operator the "wrong" ordering gives indeed the correct time evolution.

A gas of atoms in largely detuned laser light: To study the decoherence of atoms in a largely detuned laser beam we adiabatically eliminate the excited state by means of (see, e.g., Ref. [14])

$$
\hat{\Psi}_{e}(\mathbf{x}) \approx \frac{\Omega(\mathbf{x})}{\Delta} \hat{\Psi}_{g}(\mathbf{x}) \text {. }
$$

Inserting this into Eq. (12) leads to a Liouvillean

$$
\begin{array}{r}
\mathcal{L}_{g} \hat{R}=\frac{3 \gamma_{0}}{4} \int d^{3} x d^{3} x^{\prime} \frac{\Omega^{*}(\mathbf{x}) \Omega\left(\mathbf{x}^{\prime}\right)}{\Delta^{2}} J(r, \theta) \times \\
\left\{\left[\hat{R}, \hat{\rho}_{g}(\mathbf{x})\right] \hat{\rho}_{g}\left(\mathbf{x}^{\prime}\right)-\hat{\rho}_{g}(\mathbf{x})\left[\hat{R}, \hat{\rho}_{g}\left(\mathbf{x}^{\prime}\right)\right]\right\}
\end{array}
$$

with $\hat{\rho}_{g}(\mathbf{x}):=\hat{\Psi}_{g}^{\dagger}(\mathbf{x}) \hat{\Psi}_{g}(\mathbf{x})$.

Eq. (15) describes the decoherence of any Heisenberg operator acting on the ground state of the atoms in the presence of a laser beam. Its physical interpretation is that the laser beam excites an atom at position $\mathbf{x}^{\prime}$ to a virtual state. The atom then spontaneously emits a photon which is absorbed by another atom at position $\mathbf{x}$. Finally, this atom makes an induced emission of a photon into the mode of the laser beam. A simple application of Eq. 15) is the demonstration of lack of decoherence for the number density operator $\hat{\rho}_{g}(\mathbf{x})$. Since $\left[\hat{\rho}_{g}(\mathbf{x}), \hat{\rho}_{g}\left(\mathbf{x}^{\prime}\right)\right]=0$ one can immediately infer that

$$
\mathcal{L}_{g} \hat{\rho}_{g}(\mathbf{x})=0,
$$

i.e., there will be no decoherence of the number density at a point $\mathbf{x}$. This is a reasonable result since spontaneous emission should not change the population density if the atomic center-of-mass motion is neglected.

To study the phase decoherence we consider the time evolution of the field operator $\hat{\Psi}_{g}(\mathbf{x})$ itself. Introducing the operator

$$
\hat{Q}(\mathbf{x}):=\frac{3 \gamma_{0}}{4 \Delta^{2}} \int d^{3} x^{\prime} J(r, \theta) \Omega\left(\mathbf{x}^{\prime}\right) \Omega^{*}(\mathbf{x}) \hat{\rho}_{g}\left(\mathbf{x}^{\prime}\right)
$$

one can show that $\left(\mathcal{L}_{g}\right)^{n} \hat{\Psi}_{g}(\mathbf{x})=\left(\mathcal{L}_{Q}\right)^{n} \hat{\Psi}_{g}(\mathbf{x})$ with

$$
\mathcal{L}_{Q} \hat{R}:=\hat{Q}^{\dagger}(\mathbf{x}) \hat{R}-\hat{R} \hat{Q}(\mathbf{x}) .
$$

We therefore can replace $\mathcal{L}_{g}$ by $\mathcal{L}_{Q}$ for the special case of the field operator. The solution of the equation of motion $\partial_{t} \hat{R}=-\mathcal{L}_{Q} \hat{R}$ with the initial condition $\hat{R}(t=0)=$ $\hat{\Psi}_{g}(\mathbf{x})$ then can be written as

$$
\hat{R}(t)=e^{t \hat{Q}^{\dagger}(\mathbf{x})} \hat{\Psi}_{g}(\mathbf{x}) e^{-t \hat{Q}(\mathbf{x})}
$$

This expression can be further reduced by writing it in the form $\hat{R}(t)=\exp \left[-t\left(\hat{Q}-\hat{Q}^{\dagger}\right)\right] \exp [t \hat{Q}] \hat{\Psi}_{g}(\mathbf{x}) \exp [-t \hat{Q}]$. The first exponential corresponds to a unitary operator. The other two exponentials represent a non-untitary transformation of the field operator. It is easy to prove [15] that this non-unitary transformation can be reduced to

$$
e^{t \hat{Q}(\mathbf{x})} \hat{\Psi}_{g}(\mathbf{x}) e^{-t \hat{Q}(\mathbf{x})}=\exp \left[-\frac{\gamma_{0} t}{2} \frac{|\Omega(\mathbf{x})|^{2}}{\Delta^{2}}\right] \hat{\Psi}_{g}(\mathbf{x})
$$

This result just corresponds to the single-particle decoherence-effect of spontaneous emission: at point $\mathbf{x}$ the probability for the atoms to become excited is, within the adiabatic approximation, given by $|\Omega(\mathbf{x})|^{2} / \Delta^{2}$. The excited atoms then do spontaneously (i.e., incoherently) decay at a rate $\gamma_{0}$. The rate $\gamma_{0}|\Omega(\mathbf{x})|^{2} / \Delta^{2}$ therefore determines the time scale of light-induced decoherence at point $\mathbf{x}$.

It is surprising that the transformation (20) does not describe the full decoherence of the field operator but that one also has to take into account the unitary operator $U_{Q}:=\exp \left[-t\left(\hat{Q}-\hat{Q}^{\dagger}\right)\right]$. If $\hat{Q}$ is Hermitean the unitary operator becomes trivial and the total effect of decoherence reduces to that described by Eq. (20). This happens whenever the phase of the laser beam is homogeneous, for instance in a standing-wave laser field with fixed polarization. The reason is the cancellation between the contributions of counterpropagating light waves. In a running wave, however, $\hat{Q}$ is not Hermitean and manyparticle effects do appear.

To analyse the effect of the unitary operator $U_{Q}$ it is convenient to consider a fully coherent ensemble of atoms, e.g., a Bose-Einstein condensate at temperature $T=0$. In the mean-field description the condensate can be described (see, e.g., Ref. [8] by a coherent state $|\alpha\rangle=$ $\exp \left(-|\alpha|^{2} / 2\right) \exp \left(\alpha \hat{a}_{0}^{\dagger}\right)|0\rangle$, where $\hat{a}_{0}^{\dagger}=\int d^{3} x \varphi_{0}(\mathbf{x}) \hat{\Psi}_{g}^{\dagger}(\mathbf{x})$ is the creation operator for atoms in the condensate mode $\varphi_{0}(\mathbf{x})$. The number $|\alpha|^{2}$ is the number of atoms in the condensate. Our task is then to calculate the expectation value $\langle\alpha|\hat{R}(t)| \alpha\rangle$ which, after some elementary algebra, can be cast into the form

$$
\begin{aligned}
\langle\alpha|\hat{R}(t)| \alpha\rangle= & \alpha \varphi_{0}(\mathbf{x}) \exp \left[-\frac{\gamma_{0} t}{2} \frac{|\Omega(\mathbf{x})|^{2}}{\Delta^{2}}\right] \times \\
& e^{-|\alpha|^{2}}\left\langle 0\left|e^{\alpha^{*} a_{0}} e^{\alpha \tilde{a}_{0}^{\dagger}(t)}\right| 0\right\rangle,
\end{aligned}
$$

where $\tilde{a}_{0}^{\dagger}(t)$ is defined as $U_{Q} \hat{a}_{0}^{\dagger} U_{Q}^{\dagger}$. With techniques similar to that used to derive Eq. (20) one can prove that $\tilde{a}_{0}^{\dagger}(t)$ is in fact the creation operator for another mode, $\tilde{a}_{0}^{\dagger}(t)=\int d^{3} x \tilde{\varphi}_{0}(\mathbf{x}, t) \hat{\Psi}_{g}^{\dagger}(\mathbf{x})$, whose mode function is given by

$$
\begin{aligned}
& \tilde{\varphi}_{0}\left(\mathbf{x}^{\prime}, t\right)=\varphi_{0}\left(\mathbf{x}^{\prime}\right) \exp [-\frac{3}{4} i \gamma_{0} t J\left(\mathbf{x}-\mathbf{x}^{\prime}\right) \times \\
&\left.\frac{\Omega\left(\mathbf{x}^{\prime}\right) \Omega^{*}(\mathbf{x})-\Omega^{*}\left(\mathbf{x}^{\prime}\right) \Omega(\mathbf{x})}{i \Delta^{2}}\right] .
\end{aligned}
$$

Inserting this expression into Eq. (21) leads to the final result for the light-induced decoherence of a condensate at position $\mathbf{x}$, 


$$
\begin{aligned}
\langle\alpha|\hat{R}(t)| \alpha\rangle= & \alpha \varphi_{0}(\mathbf{x}) \exp \left[-\frac{\gamma_{0} t}{2} \frac{|\Omega(\mathbf{x})|^{2}}{\Delta^{2}}\right] \times \\
& \exp \left[|\alpha|^{2}\left(\int d^{3} x^{\prime} \varphi_{0}^{*}\left(\mathbf{x}^{\prime}\right) \tilde{\varphi}_{0}\left(\mathbf{x}^{\prime}, t\right)-1\right)\right] .
\end{aligned}
$$

Eq. (23) is the main result of this paper and describes the total effect of light-induced decoherence on a BoseEinstein condensate of two-level atoms. It demonstrates that there are non-local contributions to the atomic decoherence. To estimate the order-of-magnitude of these non-local terms we assume for simplicity a Gaussian condensate wave function of width $w$, i.e., $\varphi_{0}(\mathbf{x})=$ $\exp \left[-\mathbf{x}^{2} /\left(2 w^{2}\right)\right] /\left(w^{3 / 2} \pi^{3 / 4}\right)$. In addition we consider a running-wave laser field of the form $\Omega(\mathbf{x})=b \Omega_{0} \exp (i k z)$. The analysis of Eq. (23) is then reduced to a numerical evaluation of a complicated integral. To gain more physical insight we expand the exponent appearing in Eq. (22) to second order in $t$. Introducing the decoherence rate $\gamma_{D}:=\gamma_{0}\left|\Omega_{0}\right|^{2} /\left(2 \Delta^{2}\right)$ we then can write the result (23) as

$$
\begin{aligned}
\langle\alpha|\hat{R}(t)| \alpha\rangle \approx \alpha \varphi_{0}(\mathbf{x}) & \exp \left[-\gamma_{D} t\left(1+3 i N_{\lambda} A\left(z, w, k_{L}\right)\right)\right. \\
& \left.-\left(3 \gamma_{D} t\right)^{2} N_{\lambda} B\left(z, w, k_{L}\right)\right]
\end{aligned}
$$

Here $N_{\lambda}:=\left|\alpha \varphi_{0}(0)\right|^{2} \lambda_{0}^{3}$ is the number of condensed atoms in a cube of dimension $\lambda_{0}^{3}$ at the center of the condensate. $\lambda_{0}$ is the wavelength of resonant light. The functions $A\left(z, w, k_{L}\right)$ and $B\left(z, w, k_{L}\right)$ depend on the position $\mathbf{x}=z \mathbf{e}_{z}$ at which the decoherence is observed, the width $w$ of the condensate, and on the wavevector $k_{L}$ of the running laser wave. For $w=100 c / \omega_{0}$ and a detuning of $\Delta=1 \mathrm{GHz}$ they are shown in Figs. 1 and 2, respectively. $A$ only has a strong dependence on $k_{L}$ for extreme detunings above $1 \mathrm{THz}$ while $B$ is rather insensitive to the detuning.

Eq. (24) demonstrates that the additional unitary operator $U_{Q}$ does not lead to an additional exponential decay of the coherences since to first order its effect is only a position-dependent phase shift which is proportional to $A$. For longer times $\left(t \approx 1 / \gamma_{D}\right)$ the many-particle decoherence effect can become even larger than the singleparticle contribution. Since in the example given above the function $B$ is roughly proportional to the condensate density one arrives at the convincing result that the many-particle decoherence grows with the atomic density.

In conclusion, we have shown that the decoherence of a gas of ultracold atoms in a detuned laser beams is spatially varying and depends on whether the phase of the laser beam is spatially homogeneous. If this is not the case the one-particle spontaneous emission process will be accompagnied by a significant many-particle contribution with a non-exponential decay rate. This additional effect corresponds to the expectation value of the unitary operator $U_{Q}$.
Acknowledgement: I thank Jürgen Audretsch and Konstantin Krutitsky for many valuable discussions. This project has been supported by the Deutsche Forschungsgesellschaft (Forschergruppe Quantengase) and the Optik Zentrum Konstanz.

[1] S.A. Parkins and D.F. Walls, Phys. Rep. 303, 1 (1998).

[2] F. Dalfovo, S. Giorgini, L.P. Pitaevskii, and S. Stringari, Rev. Mod. Phys. 71, 463 (1999).

[3] D. M. Stamper-Kurn, M. R. Andrews, A. P. Chikkatur, S. Inouye, H.-J. Miesner, J. Stenger, and W. Ketterle, Phys. Rev. Lett. 80, 2027 (1998).

[4] K. Nemoto and F. Shibata, J. Phys. Soc. Japan 65, 3492 (1996).

[5] L. Mandel and E. Wolf, Optical coherence and quantum optics, Cambridge University Press, Cambridge 1995.

[6] K. Rzązewski and W. Zakowicz, Journ. Phys. B 25, L319 (1992).

[7] K.-P. Marzlin and W. Zhang, Eur. Phys. J. D 12, 241 (2000).

[8] W. Zhang and D.F. Walls, Phys. Rev. A 49, 3799 (1994).

[9] K.-P. Marzlin and J. Audretsch, Phys. Rev. D 57, 1045 (1998).

[10] R.H. Lehmberg, Phys. Rev. A 2, 883 (1970).

[11] Y. Castin, J.I. Cirac, and M. Lewenstein, Phys. Rev. Lett. 80, 5305 (1998).

[12] L. Santos, F. Floegel, T. Pfau, and M. Lewenstein, quantph/0007003 (2000).

[13] L. Santos, Z. Idziaszek, J.I. Cirac, and M. Lewenstein, quant-ph/0005107 (2000).

[14] K.-P. Marzlin and W. Zhang, Phys. Rev. A 57, 4761 (1998).

[15] One has to show that the left-hand and the right-hand side of Eq. (20) are the same at $t=0$ and that they fulfill the same differential equation.

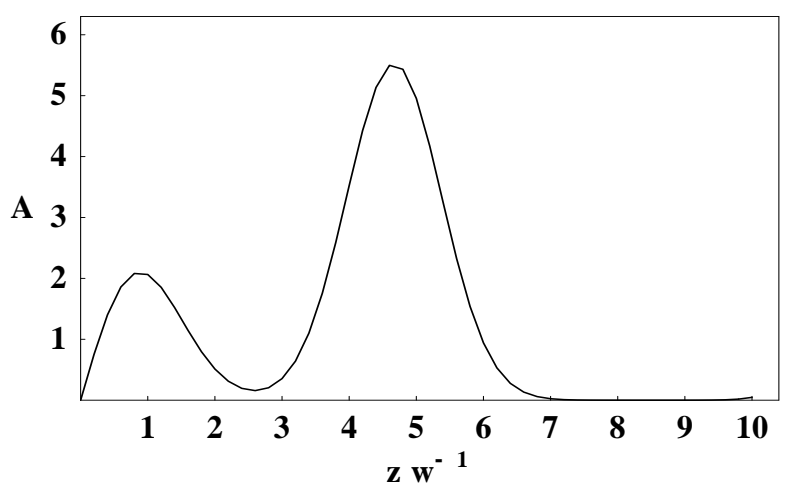

FIG. 1. The function $A\left(z, w, k_{L}\right)$, which is related to a light-induced phase shift in the condensate, as a function of the position $z$ along the z-axis. The width $w$ is $100 /(2 \pi)$ times the resonant wavelength, and the detuning was chosen to be $\Delta=1 \mathrm{GHz}$. 


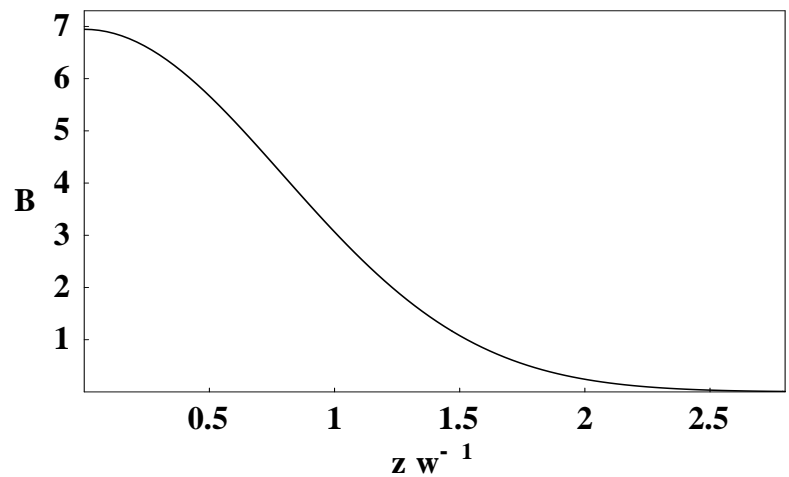

FIG. 2. The function $B\left(z, w, k_{L}\right)$, which is related to the many-particle decoherence effect, for the same parameters as in Fig. 11. 